\title{
DETECCIÓN DEL SINANAMORFO Cylindrocarpon cylindroides var. tenue SOBRE CANCROS CAUSADOS POR Neonectria fuckeliana EN Pinus radiata
}

(Detection of sinanamorph Cylindrocarpon cylindroides var. tenue on Neonectria fuckeliana cankers in Pinus radiata)

Rodrigo Morales ${ }^{1 *}$, Eduardo Molina ${ }^{2}$,Moisés Osorio ${ }^{3}$, Isabel Vives ${ }^{1}$ Cristián González², Juan Carlos Carmona²

${ }^{1}$ Universidad Austral de Chile, Facultad de Ciencias Forestales y Recursos Naturales. ${ }^{2}$ Asistente de Investigación Proyecto Fondo SAG-C6-110-NC-13. ${ }^{3}$ Ex -Académico Universidad Austral de Chile. *Autor para correspondencia: Laboratorio de Patología Forestal, Valdivia, Chile, tel.: 63-221740. e-mail: rmorales@uach.cl

RECIBID0: 05/11/2013

APROBADO: $13 / 12 / 2013$

Palabras clave: Neonectria fuckeliana (forma cylindrocarpon cylindroides var. tenue), cancros, Pinus radiata, caracterización morfológica.

Key words: Neonectria fuckeliana (cylindrocarpon cylindroides var. tenue morph), canker, Pinus radiata, morphological characterization.

\section{RESUMEN}

El presente trabajo tuvo por finalidad reportar y analizar la presencia de estructuras fungosas (esporodoquios) sobre cancros causados por Neonectria fuckeliana en árboles de Pinus radiata en Chile. Las muestras fueron colectadas en las Regiones de La Araucanía y de Los Ríos y fueron analizadas en el laboratorio. Los resultados de los análisis morfológicos y taxonómicos permitieron concluir que los esporodoquios hallados correspondían al sinanamorfo de Neonectria fuckeliana (forma cylindrocarpon cylindroides var tenue), siendo el primer reporte en el país. Estos antecedentes contribuyen al conocimiento y comprensión del ciclo patológico y epidemiológico de Neonectria fuckeliana en plantaciones de Pinus radiata.

\section{INTRODUCCIÓN}

Neonectria fuckeliana (Booth) Catlebury \& Rossman (syn. Nectria fuckeliana C. Booth) es un hongo originario del Hemisferio Norte, lugar en que se desarrolla como patógeno débil en árboles de
ABSTRACT

The present work was aimed at reporting and analyzing the presence of fungal structures (sporodochia) present on cankers caused by Neonectria fuckeliana in Pinus radiata trees in Chile. The samples were collected in the regions of $\mathrm{La}$ Araucanía and Los Ríos and analyzed in the laboratory. The results of morphological and taxonomic analysis led to the conclusion that the sporodochia found corresponded to sinanamorph of Neonectria fuckeliana (cylindrocarpon cylindroides var. tenue morph), the first report in the country. This work contribute to understanding and comprehension of the disease cycle and epidemiology of Neonectria fuckeliana on plantations of Pinus radiata.

Ios géneros Abies, Picea, Larix, y Pinus, atacando árboles debilitados y caídos. Sin embargo, en Nueva Zelanda (Hemisferio Sur) actúa como patógeno agresivo en plantaciones podadas de $P$. radiata (Peace 1962, Dick y Crane 2009). 
A fines del año 2008 se reportó por primera vez en Chile, el patógeno causante de cancros y malformaciones fustales en árboles podados de plantaciones de Pinus radiata D. Don (Morales 2009). Los primeros estudios en Chile comenzaron a realizarse desde el año 2010, investigando aspectos del ciclo biológico, distribución, y medidas relacionadas al control de este patógeno ${ }^{1}$.

Referente a los sinanamorfos señalados en la literatura para $N$. fuckeliana, se mencionan dos estados asexuales. Uno llamado Cylindrocarpon cylindroides var. tenue, el cual es nombrado como la fase asexual principal (Booth 1959, Funk 1981, Dick y Crane 2009). Y un segundo estado denominado Acremonium-like (semejante a Acremonium) y que sólo ha sido obtenido en aislamientos desde ascosporas o peritecios en medios de cultivos en laboratorio (Brayford et al. 2004, Molina et al. 2012). Actualmente, el Género Neonectria sensu lato, ha sufrido varios cambios taxonómicos debido a que especies integrantes de este Género, habían sido clasificadas en grupos por la presencia y ausencia de microconidios y clamidoporas en el pasado (Chaverri et al. 2011). Hoy día con el uso de herramientas moleculares y análisis filogenéticos, se ha determinado que varias especies integrantes del género Neonectria sensu lato, pertenecían incluso a nuevos Géneros (Rossmann et al. 2013). De esta manera, se ha establecido que Neonectria fuckeliana al igual que Neonectria coccinea, pertenecen al Grupo 1 y 4 Neonectria/ Cylindrocarpon sensu stricto (Chaverri et al. 2011).

En las Regiones de La Araucanía y de Los Ríos se detectaron sobre la corteza de los árboles afectados por $N$. fuckeliana, la presencia de esporodoquios irrumpiendo sobre los cancros, previa a la formación de los peritecio. El objetivo de este trabajo fue caracterizar y determinar los esporodoquios encontrados en los árboles afectados por $N$. fuckeliana y establecer su eventual vínculo micológico con este patógeno.

\footnotetext{
1 Proyecto Fondo SAG, «Ciclo biológico y aspectos epidemiológicos de Neonectria fuckeliana o Revirado del Pino en plantaciones de Pinus radiata. Estrategias para el control de la enfermedad».
}

\section{MATERIALES Y MÉTODOS}

\section{Colecta de muestras}

Las muestras correspondieron a pequeños trozos de corteza que presentaban esporodoquios, los cuales fueron colectados en dos plantaciones podadas de $P$. radiata de 8 y 9 años de edad respectivamente, localizadas en la Comuna de Toltén, Región de La Araucanía, y en la Comuna de Paillaco Región de Los Ríos.

Las muestras fueron analizadas en el Laboratorio de Patología Forestal de la Universidad Austral de Chile.

\section{Análisis taxonómicos de las muestras}

Para la caracterización microscópica de los esporodoquios se realizaron preparaciones y mediciones de las estructuras fúngicas bajo lupa estereoscópica y microscopio, y posteriormente las observaciones se documentaron con fotografías. Se midieron 30 micro y macroconidos considerando largo y ancho respectivamente, y se compararon con claves taxonómicas señaladas en la literatura (Booth 1959, Funk 1981, Brayfor et al. 2004).

\section{RESULTADOS}

Los esporodoquios irrumpen generando pequeñas rasgaduras sobre la corteza donde emergen hacia el exterior de ésta. Su textura es de aspecto esponjoso aunque están constituidos de micelio compacto, de color blanquecino a beige en un inicio, y a medida que éstos presentan mayor tiempo sobre la corteza, varía su tonalidad a colores naranjo pálido (Figura $1 \mathrm{~A}$ ). Posteriormente, sobre Ios esporodoquios comienzan a formarse los peritecios, correspondiente a la fase sexual de $N$. fuckeliana (Figura $1 \mathrm{~B}$ ). En un inicio los esporodoquios sobre la corteza de los árboles tienden a ser poco notorios, y son fácilmente confundidos con exudaciones de resina. Las dimensiones, en promedio, varían entre 1 a $15 \mathrm{~mm}$ de largo y 1 a 10 $\mathrm{mm}$ de ancho. En preparaciones microscópicas se puede observar gran cantidad de macroconidios, microconidios y fiálides, los que en su conjunto constituyen el esporodoquio (Figura $1 \mathrm{C}$ y D). Sobre el extremo apical de las fiálides se forman micro y macroconidios (Figura 1 D-E-F). Los microconidios son de forma oval clavado, hialinos y gutulados, y 

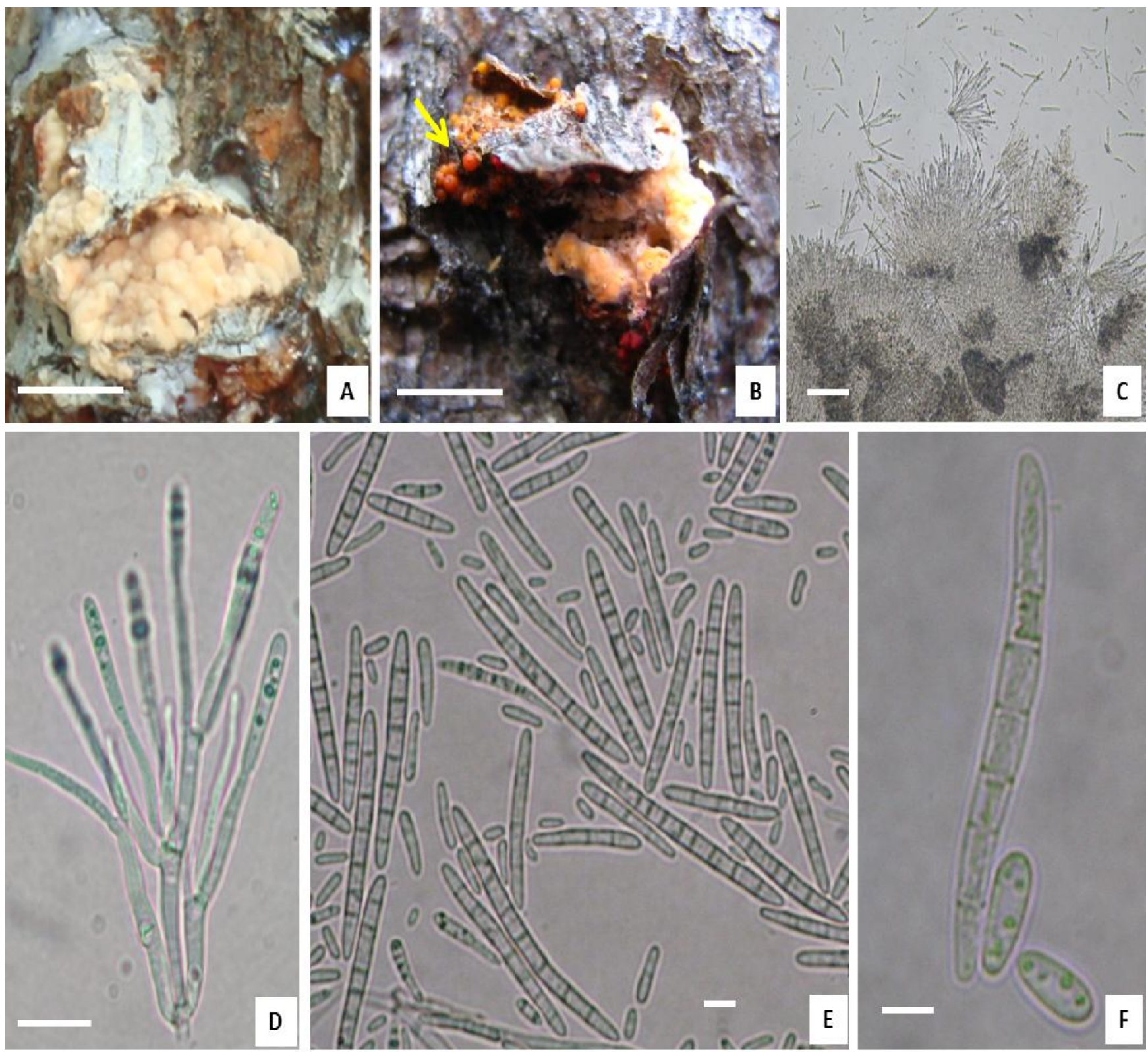

Figura 1. Características macro y microcópicas de $C$. cylindroides var tenue. en $P$. radiata A) Formación de esporodoquios sobre la corteza. B) Formación de peritecios de N. fuckeliana sobre el esporodoquio (flecha amarilla). C) Preparaciones microscópicas de esporodoquios. D) Formación de conidios desde las fiálides. E-F) Detalles microscópicos de los micro y macroconidios (Figuras A y B: barra de color blanco corresponde a $5 \mathrm{~mm}$; C: $100 \mu \mathrm{m}$; D: $50 \mu \mathrm{m}$; E y F: $10 \mu \mathrm{m}$ ).

miden entre 7,2 - 14, $4 \mu \mathrm{m}$ de largo y 2,5 - 4, $0 \mu \mathrm{m}$ de ancho (Figura $1 \mathrm{~F}$ ). Los macroconidios hialinos, de formas cilíndricas a levemente falcados y de extremos redondeados presentan en general $3,5 \mathrm{y}$ 7 septos, y sus dimensiones varían entre $30-78$ $\mu \mathrm{m}$ de largo y 3,5 - 5,2 $\mu \mathrm{m}$ de ancho (Figura $1 \mathrm{E}$ ). En las preparaciones realizadas no se evidenció la presencia de clamidosporas en el micelio.

\section{DISCUSIÓN}

Producto de este trabajo, se determinó que los esporodoquios encontrados corresponden a una especie del género Cylindrocarpon (Domsch et al. 1980, Funck 1981). La característica de los macroconidios de esta especie, son coincidente con las descrita por Booth (1959), y correspondieron a la 
especie Cylindrocarpon cylindroides var. tenue, anamorfo de Nectria fuckeliana (=Neonectria fuckeliana) asociada a cancros en especies arbóreas como Larix, Abies, Picea y Pináceas en general. Según este último autor, Cylindrocarpon cylindroides var. tenue se caracteriza por presentar de 3 a 7 septos con dimensiones de $33 \times 85 \mu \mathrm{m}$ de largo y $4 \times 7 \mu \mathrm{m}$ de ancho, concordantes con los rangos obtenidos en este trabajo. En cuanto a las dimensiones de los esporodoquios, no se mencionan mediciones al respecto, sin embargo este trabajo es coincidente con otros estudios al no presentar clamidosporas (Booth 1959, Brayford et al. 2004).

En Nueva Zelanda Dick y Crane (2009) y Crane et al. (2009) han detectado la presencia de C. cylindroides como estado anamorfo en cancros causados por $N$. fuckeliana en fustes de $P$. radiata. Sin embargo, mencionan que es un estado raramente encontrado por lo que está poco investigado en ese país.

De esta manera se concluye que los esporodoquios analizados, corresponden al sinanamorfo $C$. cylindroides var. tenue, fase asexual descrita para $N$. fuckeliana de acuerdo a los antecedentes bibliográficos consultados. Aunque algunos autores como Brayford et al. (2004), mencionan que la asociación entre Neonectria fuckeliana (syn. Nectria fuckeliana) y C. cylindroides no sería correcta (según análisis moleculares y obtención de la fase asexual semejante a Acremoniumen en cultivos). Castlebury et al. (2006) rebate dicha conclusión, señalando que es correcta, y que Brayford et al. (2004) se equivocó en los análisis moleculares, habiendo utilizado erróneamente secuencias de ADN de Fusarium solani. Chaverri et al. (2011) y Rossmann et al. (2013) ratifican la relación Neonectria/Cylindrocarpon sensu stricto a través de estudios filogenéticos con herramientas moleculares, lo cual lleva inequívocamente a establecer que $N$. fuckeliana tiene como fase asexual $C$. cylindroides.

En este trabajo se ratifica la relación in situ de Neonectria/Cylindrocarpon entre ambos estados y a través de análisis morfológicos en laboratorio. Sería recomendable a futuro, ratificar a nivel de cultivos dicha relación y empleando herramientas moleculares.
En nuestro trabajo nos hemos basado en los recientes cambios referentes a los hongos polimórficos del nuevo «International Code of Nomenclature for algae, fungi, and plants (ICN)» y en el Artículo 59.1, McNeill et al. 2013. Que estipula el empleo de un solo nombre científico para cada especie de hongo desde enero 2013, bajo un consenso nacional de expertos.

\section{CONCLUSIONES}

De los análisis realizados se identificó Neonectria fuckeliana (forma cylindrocarpon cylindroides var. tenue) asociado a cancros en árboles de $P$. radiata. Este reporte corresponde al primer hallazgo de este sinanamorfo de N. fuckeliana en el Chile.

\section{AGRADECIMIENTOS}

Este estudio fue desarrollado y financiado en el marco del proyecto FONDO SAG C6-110-NC13 «Ciclo biológico y aspectos epidemiológicos de Neonectria fuckeliana o Revirado del Pino en plantaciones de Pinus radiata. Estrategias para el control de la enfermedad».

\section{REFERENCIAS}

Brayford, D.; Honda, B.; Mantiri, B. \& Samuels, G. (2004). Neonectria and Cylindrocarpon: The Nectria mammoidea group and species lacking microconidia. Mycologia 96(3): 572-597.

Booth, C. 1959. Studies of Pyrenomycetes IV. Nectria (part 1). Mycological Papers 73: 56-57. MycoBank, Fungal Databases, Nomenclature and Species Banks, http://www.mycobank.org/Biolomics.aspx? Table $=$ Mycobank\&MycoBankNr_ $=301766$

Castlebury, L. A.; Rossman, A. Y.; \& Hyten, A. S. (2006). Phylogenetic relationships of Neonectria/ Cylindrocarpon on Fagus in North America. Canadian Journal of Botany 84: 1417-1433.

Chaverri, P.; Salgado, C.; Hirooka, Y.; Rossman, A.; Samuels, G. (2011). Delimitation of Neonectria and Cylindrocarpon (Nectriaceae, Hypocreales, Ascomycota) and related genera with Cylindrocarponlike anamorphs. Studies in Mycology 68:57-78. 
Crane, P. E.; Hopkins, A. J. M.; Dick, M. A.; \& Bulman, L. S. (2009). Behaviour of Nectria fuckeliana causing a pine canker disease in New Zealand. Canadian Journal of Forest Research 39: 2119-2128.

Dick, M. A. \& Crane, P. E. (2009). Neonectria fuckeliana is pathogenic to Pinus radiata in New Zealand. Australasian Plant Disease Notes 4: 12-14.

Domsch, K.; Gams, W. \& Anderson, T. (1980). Compendium of soil fungi. USA. Academic Press. v. 1. $859 \mathrm{p}$.

Funk, A. (1981). Parasitic Microfungi of western trees. Canada. Canadian Forestry Service Pacific Forest Research Centre Victoria. $164 \mathrm{p}$.
Molina, E.; Morales, R.; Valenzuela, E. \& Vives, V. (2012). Caracterización morfológica de Acremonium sp. asociado a Neonectria fuckeliana en Pinus radiata en Chile. Bol. Micol. 27(2):32-38.

Morales, R. (2009). Detección de Neonectria fuckeliana en Chile, asociado a cancros y malformaciones fustales en plantaciones de Pinus radiata. Bosque 30(2):106-110.

Rossman, A.; Seifert, K.; Samuels, G.; Minnis, A.; Schroers, H.; Lombard, L.; Crous,P.; Põldmaa, K.; Cannon, P.; Summerbell, R.; Geiser, D.; Zhuang, W.; Hirooka, Y.; Herrera, C.; Salgado-Salazar, C.; \& Chaverri, P. (2013). Bionectriaceae, Hypocreaceae, and Nectriaceae (Hypocreales) proposed for acceptance or rejection. IMA Fungus 4(1): 41-51.

\section{CONFLICTO DE INTERESES}

Los autores declaran no tener conflicto de intereses. 